\title{
Editorial
}

\section{Extracellular vesicles as novel biomarkers and pharmaceutic targets of diseases}

\author{
Yanfang $\mathrm{CHEN}^{1, *}$, Yaoliang TANG ${ }^{2}$, Guo-Chang FAN ${ }^{3}$, Dayue Darrel DUAN ${ }^{4}$ \\ ${ }^{1}$ Department of Pharmacology \& Toxicology, Boonshoft School of Medicine, Wright State University, Dayton, OH 45435, USA; \\ ${ }^{2}$ Department of Medicine, Vascular Biology Center, Medical College of Georgia, Augusta University, Augusta, GA 30912, USA; \\ ${ }^{3}$ Department of Pharmacology and Cell Biophysics, University of Cincinnati College of Medicine, Cincinnati, OH 45267, USA; \\ ${ }^{4}$ Department of Pharmacology, University of Nevada School of Medicine, Reno, NV 89557, USA
}

Acta Pharmacologica Sinica (2018) 39: 499-500; doi: 10.1038/aps.2018.15

Extracellular microvesicles (MVs) and exosomes (EXs) are the major types of extracellular vesicles (EVs) which can carry and transfer molecular messages (proteins, mRNAs and microRNAs, etc $)^{[1]}$. Thereby, EVs could serve to mark the changes of the producing cells and to function on the recipient cells ${ }^{[2]}$. These features deem EVs as potential biomarkers and pharmacological targets for various diseases including metabolic, vascular and neurological diseases and tumors ${ }^{[3]}$. The current special feature provides reviews and original research articles on novel pharmacological studies of MVs and EXs.

Samanta $e a l^{[4]}$ provided a comprehensive review on the classification of EVs, biogenesis and composition of EXs. This review focuses on the functional usage of EXs in therapy, and the potential advantages and insufficiencies for therapeutic purposes. The biological and pathological functions of EXs in cardiovascular disease, diabetes, neurodegenerative disease, liver inflammation and cancers have been covered in this article. EXs containing aggregation-prone proteins involved in Parkinson's disease, Alzheimer's disease, and amyotrophic lateral sclerosis have all been found in the cerebral spinal fluid and blood of patients affected by these disorders. The authors also highlighted the different strategies such as targeting the formation, release, uptake, and trafficking of EVs to attenuate adverse functions of EXs during diseases. Li et al ${ }^{[5]}$ provided a review with focuses on the ESCRT-independent regulation of cargo loading into EXs including lipid raft and ceramide mediated mechanisms, as well as the reported EXs or EX-mimics with therapeutic effects. Modification of cargo can confer additional merits such as enhanced effects and targeting capability on EXs.

Tremendous effort has been spent to elucidate the versatile

\footnotetext{
*To whom correspondence should be addressed.

E-mail yanfang.chen@wright.edu
}

roles of mammalian cell-released EVs in the process of human disease and injury repair. However, bacterium-released membrane vesicles have been paid less attention, especially their interaction with mammalian cells and their eventual impact on human health and disease. In this special feature, $\mathrm{Yu}$ et $\mathrm{al}^{[6]}$ highlighted recent findings on the biogenesis and characterization of Gram-negative bacterium-released outer membrane vesicles (OMVs) and Gram-positive bacterium-released membrane MVs, interactions of OMVs/MVs with mammalian cells and pathological/beneficial effects of OMVs/MVs on infectious diseases. This review also discusses how to engineer these bacterial MVs as therapeutic agents for the treatment of human diseases.

EXs can modulate target cell differentiation through their carried microRNAs (miRs). Embryonic stem cells (ESCs) derived from the blastocyst of the early embryo could provide a source of hematopoietic stem cells (HSCs). Previous studies have shown that microRNA-126 (miR-126) is highly expressed in HSCs and plays a role in hematopoiesis. Liao et al ${ }^{[7]}$ reported that conclusion, HSC-EXs can promote hematopoietic differentiation of ESCs through modulating the miR126/ Notch1 pathway. This study suggests a novel approach to increase the production of HSCs for clinical use.

The ceramide pathway has been suggested to trigger the secretion of tumor-suppressive miRs. Qian et al ${ }^{[8]}$ determined whether the ceramide pathway regulates the cellular and exosomal miRs of multiple myeloma (MM) and analyzed the possible links of caspase-dependent pathway and tumor-suppressive miRs (miR-202, miR-16, miR-29b and miR-15a). Their data indicate that ceramide pathway modulates MM survival probably via the caspase pathway and exosomal miR mechanisms directly and indirectly, which might be developed as a novel therapeutic avenue for MM. Sun et a $l^{[9]}$ gave a brief review on EXs as a double-edged sword in tumor therapy. 
EXs may contribute in invasion and metastasis of cancer cells by interacting with the tumor microenvironment. The signaling pathways modulated by EXs could be the new targets for the treatment of cancer.

It is interesting that a traditional Chinese medicine, Suxiao Jiuxin Pill (SJP), was found to be able to modulate cardiac mesenchymal stem cells (C-MSC) derived EXs and lead to epigenetic chromatin remodeling in recipient cardiomyocytes. As reported by Ruan et al ${ }^{[10]}, \mathrm{SJP}$, which is comprised of two principal components, tetramethylpyrazine (TMP) and borneol (BOR), increases H3K27me3 levels in EX treated cardiomyocytes by significantly reducing the expression of the H3K27 demethylase UTX with minimal effects on expression of all three H3K27 methylases (EZH1, EZH2 and EED) and the H3K27 demethylase JMJD3 in EX treated cells. For the first time in the literature, this study highlights the novel role of stem cell-derived EXs to epigenetically remodel chromatin in recipient cells. Furthermore, they also showed that SJP promotes EX secretion from C-MSC by upregulating Rab27a and Rab27b GTPases, consistent with a novel mechanism for SJP in modulating cardiac homeostasis ${ }^{[11]}$.

Although accumulated evidence strongly support that EXs and MVs could be used as biomarkers and novel therapeutic approaches for various diseases, further in-depth exploration of effective technologies and protocols for EV isolation, purification, and detection of EVs with various origins and better understanding of the dynamic regulation and detailed functional roles of EVs in the context of health and disease remain to be accomplished before EV-based biomarkers and therapeutic interventions become available for clinical use. We hope that the original research articles and review articles presented in this special feature represent the current advances in this field and would contribute to the future development of this promising tool for diagnosis and therapy of human diseases.

\section{References}

1 Merchant ML, Rood IM, Deegens JKJ, Klein JB. Isolation and characterization of urinary extracellular vesicles: implications for biomarker discovery. Nat Rev Nephrol 2017; 13: 731-49.

2 Ratajczak MZ, Ratajczak J. Extracellular microvesicles as game changers in better understanding the complexity of cellular interactions: from bench to clinical applications. Am J Med Sci 2017; 354: 449-52.

3 Ying W, Riopel M, Bandyopadhyay G, Dong Y, Birmingham A, Seo JB, et al. Adipose tissue macrophage-derived exosomal miRNAs can modulate in vivo and in vitro insulin sensitivity. Cell 2017; 171: 372-84.

4 Samanta S, Rajasingh S, Drosos N, Zhou Z, Dawn B, Rajasingh J. Exosomes: new molecular targets of diseases. Acta Pharmacol Sin 2018; 39: 501-13.

5 Li SP, Lin ZX, Jiang XY, Yu XY. Exosomal cargo-loading and synthetic exosome-mimics as potential therapeutic tools. Acta Pharmacol Sin 2018; 39: 542-51.

6 Yu YJ, Wang XH, Fan GC. Versatile effects of bacterium-released membrane vesicles on mammalian cells and infectious/ inflammatory diseases. Acta Pharmacol Sin 2018; 39: 514-33.

7 Liao FL, Tan L, Liu H, Wang JJ, Ma XT, Zhao B, et al. Hematopoietic stem cell-derived exosomes promote hematopoietic differentiation of mouse embryonic stem cells in vitro via inhibiting the miR126/Notch1 pathway. Acta Pharmacol Sin 2018; 39: 552-60.

8 Cheng Q, Li X, Wang Y, Dong M, Zhan FH, Liu J. The ceramide pathway is involved in the survival, apoptosis and exosome functions of human multiple myeloma cells in vitro. Acta Pharmacol Sin 2018; 39: 5618.

9 Sun W, Luo JD, Jiang H, Duan DD. Tumor exosomes: a double-edged sword in cancer therapy. Acta Pharmacol Sin 2018; 39: 534-41.

10 Ruan XF, Li YJ, Ju CW, Shen Y, Lei W, Chen C, et al. Exosomes from Suxiao Jiuxin pill-treated cardiac mesenchymal stem cells decrease H3K27 demethylase UTX expression in mouse cardiomyocytes in vitro. Acta Pharmacol Sin 2018; 39: 579-86.

11 Ruan XF, Ju CW, Shen Y, Liu YT, Kim IM, Yu H, et al. Suxiao Jiuxin pill promotes exosome secretion from mouse cardiac mesenchymal stem cells in vitro. Acta Pharmacol Sin 2018; 39: 569-78. 